Revue internationale d'éducation de Sèvres

33 | septembre 2003

L'enseignement des langues vivantes à l'étranger : enjeux et stratégies

\title{
Des efforts non récompensés. Le cas de la Suède
}

Unrewarded efforts - the Swedish experience

Esfuerzos no recompensados. El caso de Suecia

\section{Béatrice Cabau-Lampa}

\section{OpenEdition \\ Journals}

Édition électronique

URL : http://journals.openedition.org/ries/1640

DOI : $10.4000 /$ ries. 1640

ISSN : 2261-4265

Éditeur

Centre international d'études pédagogiques

Édition imprimée

Date de publication : 1 septembre 2003

ISBN : 978-2-85420-557-1

ISSN : $1254-4590$

Référence électronique

Béatrice Cabau-Lampa, "Des efforts non récompensés. Le cas de la Suède ", Revue internationale d'éducation de Sèvres [En ligne], 33 I septembre 2003, mis en ligne le 23 novembre 2011, consulté le 19 avril 2019. URL : http://journals.openedition.org/ries/1640 ; DOI : 10.4000/ries.1640

(c) Tous droits réservés 


\section{Des efforts non récompensés}

\section{Le cas de la Suède}

\section{Béatrice Campau-Labat}

Il y a maintenant plus de quarante ans, en 1962 plus précisément, l'apprentissage de l'anglais devenait obligatoire en Suède pour tous les élèves de la toute nouvelle école obligatoire (école de base, grundskola) d'une scolarité de neuf années. Cette mesure était présentée comme une étape cruciale dans le processus de démocratisation de la société suédoise ${ }^{1}$. Cette date représente également une étape essentielle pour le français, lequel était dorénavant mis sur un pied d'égalité avec l'allemand en tant que seconde langue étrangère optionnelle, alors que la langue germanique avait été la première langue étrangère enseignée pendant plusieurs décennies. Les mesures prises allaient réaffirmer l'importance attachée à la formation linguistique des jeunes Suédois.

\section{UN RENFORCEMENT DE L'ENSEIGNEMENT DES LANGUES}

Au début des années quatre-vingt-dix, la Suède est entrée dans une profonde récession au moment même où elle préparait son entrée dans l'Union européenne qui se matérialiserait en 1995. Lorsqu'un gouvernement de coalition de droite a pris le pouvoir en 1991, le nouveau ministre de l'Éducation a mis en avant la nécessité de renforcer l'enseignement des langues dans le cadre scolaire. Cette volonté a été prise en considération lors de l'élaboration du nouveau plan d'enseignement de 1994, le Lpo94, qui a été introduit dans toutes les écoles obligatoires à l'automne 1997/98. La principale raison en était que le nombre d'élèves ayant appris une langue étrangère autre que l'anglais à la fin du lycée était considéré comme trop faible. Cela a incité les responsables suédois de l'éducation à instaurer l'apprentissage obligatoire d'une seconde langue étrangère ${ }^{2}$. Le taux élevé d'abandons en cours d'apprentissage de seconde langue étrangère était dû au fait que les élèves faisaient (et font encore) un choix tactique, considérant qu'il est plus facile d'obtenir une meilleure note dans une matière non linguistique. Il est bon ici de mentionner que les résultats scolaires sont déterminants pour l'entrée dans l'enseignement supérieur.

1. SOU (1948), 1946 års Skolkommissions betänkande med förslag till riktlinjer för det svenska skolväsendets utveckling (Rapport de la Commission scolaire de 1946 avec proposition d'orientation pour le développement de l'instruction publique suédoise), SOU 1948: 27, Ecklesiastikdepartementet.

2. Statens Offentliga Utredningar (1992), Skola för bildning. Betänkande av läroplanskommittén (L’école pour l'éducation. Réflexions du Comité du plan d'enseignement), SOU 1992: 94, Stockholm. 
Selon le Lpo94, tous les élèves de sixième ou septième année doivent choisir une matière linguistique, à savoir: une seconde langue étrangère, la langue des signes, le suédois ou l'anglais renforcé. Les élèves d'origine immigrée peuvent également choisir entre leur langue maternelle et le suédois langue seconde. Chaque école doit proposer au moins deux des trois langues instituées «langues B» (pour désigner les secondes langues étrangères), à savoir l'allemand, le français et l'espagnol, ce dernier étant dorénavant placé sur un pied d'égalité avec les deux autres langues. La durée de chaque cours a été portée à soixante minutes contre quarante jusqu'alors. Une commune se trouve dans l'obligation de mettre en place l'enseignement d'une langue B sous deux conditions ${ }^{3}$ : il faut un minimum de cinq élèves désirant étudier la langue en question et les élèves doivent avoir la possibilité de poursuivre cet apprentissage au lycée. La première condition ne s'applique pas dans le cas où le choix de l'élève se porte sur le same (lapon) ou le finnois (qui ont été reconnus comme langues minoritaires nationales en $2000^{4}$ ). L'enseignement d'une troisième langue étrangère (langue C) peut également être proposé aux élèves qui le souhaitent en dernière année de l'école de base, selon les conditions citées ci-dessus. Ce dernier s'effectue dans le cadre du «choix de l'élève» ou dans celui du «choix de l'école» (skolans val), temps alloué à chaque école pour définir un profil de son choix, s'élevant respectivement à 470 et 410 heures sur l'ensemble de la scolarité effectuée à l'école de base.

Le nombre de cours consacrés à l'apprentissage d'une seconde langue à l'école de base a augmenté de $25 \%$. Le nombre de cours d'anglais reste, lui, presque inchangé. C'est à chaque établissement de décider, là encore, quand cet apprentissage doit débuter. En 2001/02, 33,4\% des élèves ont ainsi choisi de mettre à profit cette nouvelle possibilité en commençant à apprendre cette langue dès la première année de scolarité, alors qu'auparavant l'apprentissage de l'anglais débutait généralement en quatrième année, et pour 38,4\% d'entre eux, les cours d'anglais commencent en troisième année scolaire ${ }^{5}$. Il est à noter que la Confédération générale du Travail (LO, Landsorganisationen) avait même exprimé dès la fin des années 1960 le désir que cet apprentissage soit obligatoire dès les première et seconde années de la scolarité ${ }^{6}$.

3. Svensk författningssamling, SFS 1994: 1194, "Grundskoleförordning» ("Règlement de l'école de base»), chapitre $2, \S 7$.

4. Outre ces deux langues, la Suède a également reconnu le finnois de la vallée-frontière du fleuve Torne dans le nord du pays, le romani et le yiddish.

5. Barnomsorg, skola och vuxenutbildning i siffror, Skolverkets rapport $\mathrm{nr} 214,2001$. Les chiffres qui suivent ont été calculés d'après cette publication.

6. Sutton G. (1979), «Cultural and Socio-economic Factors in the Formation of Foreign Language Education Policy in Sweden - with a comparison with the Finnish Case », Language Problems and Language Planning, vol. 3. 


\section{LE DÉBAT AUTOUR DE L'APPRENTISSAGE OBLIGATOIRE DE DEUX LANGUES}

Lors de l'élaboration du plan d'enseignement de 1980, il avait été déjà question de savoir si l'apprentissage d'une seconde langue étrangère devait être obligatoire ou totalement supprimé pour se conformer à la logique de l'école unique. Il était à l'époque jugé irréaliste d'imposer cet apprentissage. Mais, comme les représentants du monde de l'industrie et du commerce protestaient énergiquement contre sa suppression à l'école de base, le compromis trouvé a consisté à préserver le système d'option alors existant qui faisait de l'allemand et du français des matières facultatives ${ }^{7}$. D'après une enquête effectuée durant l'année scolaire 1987/88 auprès d'enseignants de français et d'allemand, il ressortait que la majorité d'entre eux $(62 \%)$ se prononçaient contre cette suggestion ${ }^{8}$.

Si, lors du débat qui s'est déroulé dans le cadre de l'élaboration du Lpo $94^{9}$, on a pu assister cette fois-ci à une unanimité concernant le renforcement de l'enseignement linguistique, le point de discorde a porté sur l'année où l'apprentissage de l'anglais et de la seconde langue étrangère devait commencer. Plusieurs voix se sont élevées contre la proposition du Comité d'enseignement selon laquelle les cours d'anglais devraient commencer dès la première année scolaire et ceux de langue $B$ dès la sixième. Les arguments étaient d'ordre pédagogique, pratique et financier. Selon certains, imposer l'apprentissage de l'anglais dès la première année de la scolarité mettait en danger l'apprentissage correct du suédois, considéré comme prioritaire. Ils attiraient l'attention sur les dépenses qu'occasionnerait cet apprentissage précoce au niveau de la formation continue des enseignants. On chercha donc à trouver un compromis. Là encore, la décentralisation joua pleinement son rôle puisqu'il revint désormais à chaque commune de décider quand l'apprentissage de l'anglais et de la seconde langue étrangère devait commencer.

\section{LA RÉFORME DE 1995}

Nous pouvons étudier les résultats de la réforme introduite en 1995 à l'aide du tableau suivant ${ }^{10}$. L'objectif d'augmenter le nombre d'élèves apprenant une seconde langue a été atteint à l'école obligatoire: en 2001/02, plus de $63 \%$ des élèves de neuvième suivaient des cours d'allemand, de français, d'espagnol ou autres, soit une augmentation d'environ $10 \%$ depuis le milieu des

7. Tornberg U., Sörensen C. (1985), Tyska, Metodboks serien, Stockholm, Liber Utbildningsförlaget, p. 13.

8. SÖ (1991), Undervisningen i franska och tyska i grundskolan, R 91:12, Stockholm.

9. Voir les propositions gouvernementales 1992/93:220 et 1992/93:250, Annexes 1 et 2.

10. Tableau réalisé à partir des statistiques publiées par Skolverket dans Sveriges officiella statistik Barnomsorg, skola och vuxenutbildning i siffror. 
années 1990. Cette augmentation est principalement due à la popularité de la langue hispanique: de nos jours, un élève sur cinq en sixième l'étudie, contre seulement $3,5 \%$ en $1995 / 96$. Il semble se poser comme un sérieux concurrent du français: en effet, alors qu'il était appris par $11 \%$ des élèves de dernière année de l'école de base en 2001/02, le français ne le distançait de quelques points seulement, à savoir $19 \%$. Le pourcentage d'élèves commençant l'apprentissage d'une langue $B$ - en règle générale, l'allemand, le français ou l'espagnol - une année plus tôt, en sixième, a augmenté depuis la mise en place du Lpo94: $60,5 \%$ en 1996/97 contre près de $70 \%$ en 2001/02. C'est toujours l'option de l'allemand qui remporte le plus de succès auprès des élèves: en 2001, elle a été choisie par plus de $34 \%$ des élèves de septième contre $22 \%$ pour le français. Mais sa popularité décline ces dernières années.

Le taux d'abandon reste élevé, quelle que soit la langue concernée: sur environ $79 \%$ des élèves suivant l'apprentissage d'une seconde langue en septième en 1999, il n'y avait plus en 2001/02 que $63 \%$ en neuvième année. Un tiers des élèves avait choisi une autre option pour remplacer ces cours. Un élève sur cinq (la plupart du temps un garçon), abandonne cet apprentissage, souvent

Option linguistique choisie de la $6^{\mathrm{e}}$ à la $9^{\mathrm{e}}$ année à l'école de base entre 1992 et 2001 (en \%)

\begin{tabular}{|c|c|c|c|c|c|c|c|c|c|c|}
\hline & 1992 & 1993 & 1994 & 1995 & 1996 & 1997 & 1998 & 1999 & 2000 & 2001 \\
\hline CLASSE 6 / LANGUE B & & & & 50,5 & 60,8 & 68 & 69,4 & 70 & 70 & 69,9 \\
\hline français & & & & 16,6 & 18,5 & 20,7 & 20,8 & 19,7 & 18,8 & 17,1 \\
\hline allemand & & & & 30 & 33 & 34,1 & 32,8 & 30 & 27,4 & 24,1 \\
\hline espagnol & & & & 3,6 & 5,8 & 8,8 & 10,7 & 13,3 & 16,8 & 20,9 \\
\hline autres & & & & 0,3 & 3,5 & 4,4 & 5,1 & 7,0 & 7,0 & 7,8 \\
\hline CLASSE 7/LANGUE B & 69,6 & 68 & 66,4 & 80,5 & 79,6 & 80,1 & 79,1 & 78,9 & 79,2 & 79,1 \\
\hline français & 16,9 & 19,6 & 20,9 & 22,7 & 23,9 & 24 & 24,3 & 24,1 & 22,6 & 22,1 \\
\hline allemand & 50,9 & 46,2 & 42,4 & 51,4 & 49,4 & 47,2 & 43,4 & 40,5 & 38 & 34,4 \\
\hline espagnol & & & & 6,2 & 6,0 & 8,7 & 11,2 & 14,2 & 18,3 & 22,4 \\
\hline autres & 1,8 & 2,1 & 3,1 & 0,2 & 0,4 & 0,2 & 0,2 & 0,1 & 0,3 & 0,2 \\
\hline CLASSE 8/LANGUE B & 58,9 & 60,9 & 58.8 & & 72,7 & 71,4 & 71,4 & 70,9 & 70,8 & 71,1 \\
\hline français & 11,1 & 14,8 & 16,7 & 18,2 & 20,6 & 21,5 & 21,4 & 22 & 21,6 & 20,4 \\
\hline allemand & 46,0 & 44,1 & 39,5 & 37,0 & 46,3 & 44,3 & 42,1 & 38,8 & 36,2 & 33,7 \\
\hline espagnol & 2,0 & 5,5 & 5,4 & 7,5 & 10 & 12,7 & 16,7 & & & \\
\hline autres & 1,8 & 2,0 & 2,6 & $?$ & 0,3 & 0,2 & 0,4 & 0,1 & 0,3 & 0,3 \\
\hline CLASSE 9/LANGUE B & 50,6 & 52,3 & 53,2 & $\sim$ & $\sim$ & 64,8 & 63,3 & 64 & 63,4 & 63,2 \\
\hline français & 13,3 & 10,2 & 13,1 & 15,1 & 16,2 & 18,5 & 19,1 & 19,3 & 19,8 & 19,4 \\
\hline allemand & 35,8 & 40,5 & 37,9 & 35,1 & 32,8 & 41,2 & 39,2 & 37,6 & 34,4 & 32,2 \\
\hline espagnol & 1,1 & 1,7 & 4,8 & 4,7 & 6,7 & 8,9 & 11,3 & & & \\
\hline autres & 1,5 & 1,6 & 2,2 & $?$ & $?$ & 0,3 & 0,3 & 0,4 & 0,3 & 0,3 \\
\hline
\end{tabular}


pour apprendre une autre langue au lycée. Et à la sortie de l'école obligatoire, on observe une réduction du nombre d'élèves ayant obtenu une note dans une langue $\mathrm{B}^{11}$.

Il semble qu'il soit difficile de motiver les élèves quant à l'importance de poursuivre jusqu'au bout leurs études de seconde langue étrangère. Dès que le niveau des connaissances attendu devient trop difficile à atteindre, les élèves abandonnent les cours de langue B pour opter pour des cours de soutien de suédois ou d'anglais ${ }^{12}$. Ils invoquent également d'autres raisons: il n'est pas nécessaire de connaître une autre langue que l'anglais; ils ont peu l'occasion de s'exprimer oralement durant les cours; et enfin les professeurs changent souvent en cours d'année. Les professeurs, quant à eux, déplorent que les élèves soient moins persévérants qu'autrefois, tout en admettant qu'apprendre une langue exige de gros efforts de la part des élèves ${ }^{13}$.

Les chiffres concernant l'enseignement secondaire ne font que renforcer ce constat: le pourcentage de lycéens ayant obtenu une note finale en langue B (allemand, français ou espagnol) ne s'élevait qu'à $29 \%$ en 2001/02, soit une baisse de $3 \%$ par rapport à l'année précédente ${ }^{14}$. La conséquence en est qu'à l'université, des cours pour débutants doivent désormais être organisés pour les langues les plus courantes. Cela ne semble pas inquiéter outre mesure le comité parlementaire chargé d'étudier la restructuration future de l'enseignement secondaire, car il n'a consacré que quelques lignes aux langues sur un rapport de plus de 500 pages ${ }^{15}$. Pourtant, le ministre de l'Éducation avait pris position sur l'étude des langues au Parlement en juin 2001 en déclarant «alarmant» l'intérêt décroissant des jeunes Suédois pour l'apprentissage des langues ${ }^{16}$.

\section{NOUVEAUX PLANS DE COURS}

Le renforcement de l'enseignement des langues introduit par le Lpo94 ne s'est pas accompagné de mesures correspondantes à l'école secondaire. Avec la réforme des plans de cours de 2000, un nouveau modèle de description des objectifs à atteindre pour l'apprentissage linguistique a été introduit sur la base de sept niveaux (contre six dans le Cadre européen commun de référence pour les langues). Par souci de continuité, il s'applique aussi bien à l'école obligatoire qu'au lycée avec le premier niveau correspondant au niveau débutant et le dernier au plus haut niveau pouvant être atteint au lycée. Ce nouveau système

11. Malmberg P., De moderna språken i grundskolan och gymnasieskolan från 1960 och framåt, Skolverket, Dnr 99:610, 2000. http://www.skolutveckling.se/utvecklingsteman/sprak/filer/historik.pdf

12. Sörensen C., Språkvalet i grundskolan-en pilotundersökning, Skolverket, Stockholm, 2000.

13. Article du Dagens Nyheter «Allt fler elever struntar i språk», 16 février 2003.

14. http://www.skolverket.se/pdf/gy_slutbetyg_analys.pdf

15. SOU 2002:120, Åtta vägar till kunskap - En ny struktur för gymnasieskolan, Gymnasiekommitténs slutbetänkande. Stockholm, Fritzes.

16. Snabbprotokoll 2001/02:118, 3 juni 2001, Svar på interpellation 2001/02:454 om språkstudier i skolan, http://www.riksdagen.se/debatt/0102/prot/118/HTFRAME.HTM 
de structure uniforme est supposé faciliter une progression plus claire d'un niveau à l'autre et la comparaison au niveau international ${ }^{17}$. A l'école de base, le niveau quatre correspond à la neuvième année pour l'anglais et pour les langues «modernes» (nouvelle désignation des langues B), seuls existent les deux premiers niveaux. Les niveaux cinq, six et sept pour les cours de langues modernes au lycée n'existaient pas dans l'ancien système. Chaque niveau est défini selon les objectifs à atteindre par l'élève, lesquels sont identiques pour l'anglais et la seconde langue étrangère. En neuvième année, des critères d'évaluation pour les notes «bien» et «très bien» sont définis en anglais niveau quatre et en seconde langue niveaux un et deux. Dans la mesure où le niveau deux des langues modernes est un peu moins élevé que celui décrit dans l'ancien plan de cours, les élèves sont censés avoir plus de temps pour atteindre les objectifs d'apprentissage, ce qui pourrait les inciter à choisir plus volontiers une seconde langue étrangère. Au lycée, les critères existent pour les notes "passable», «bien» et «très bien» pour tous les cours de langue. Chaque module de langue équivaut à cent crédits et, dans le programme de sciences sociales, une filière "langues» de quatre cents crédits a été créée.

Depuis 2000, l'accent est davantage mis sur la réflexion à mener par les élèves sur leur propre apprentissage de la langue et leur responsabilité dans ce domaine. Par ailleurs, les élèves doivent être continuellement appelés à effectuer un travail de réflexion sur les similarités et les différences existant entre les cultures du/des pays de langue cible et leurs propres expériences. Les principes fondamentaux de compétence communicative (dans laquelle entrent les fonctions de réception, d'interaction et de production) et de compréhension interculturelle ont été préservés. La Suède suit depuis longtemps déjà les travaux du Conseil de l'Europe. Elle a accepté le concept d'éducation interculturelle comme base de contenu et de méthodes de travail à l'école par la décision parlementaire du 28 février 1985 (dans le cadre de l'éducation pour enfants de migrants ${ }^{18}$ ).

Le nouveau plan de cours pour l'anglais de $2000{ }^{19}$ présente cette langue comme jouant un rôle essentiel dans l'école suédoise. Dans celui pour les langues modernes, le fait de pouvoir parler plusieurs langues est présenté comme important dans le contexte des contacts internationaux, de l'internationalisation du marché du travail, du développement des techniques d'information et de communication et dans la perspective d'études futures. Ces deux apprentissages visent à préserver et à développer le goût des langues chez les élèves ainsi que leur compétence pour apprendre une langue. Un modèle suédois du portfolio européen des langues a été élaboré par le département de la formation continue de l'univer-

17. Skolverket, Nya kursplaner i språk, Nyhetsbrev, 20 december 1999, Nr 20.

18. Cabau-Lampa B., «Home Language instruction in Sweden: what is the situation in 1998 », European Journal of Intercultural Studies, Vol.10, No1, 1999 et «L'expérience suédoise en matière d'enseignement des langues-cultures d'origine », Language Problems \& Language Planning, Vol. 24, Nr 2, 2000.

19. Grundskolans kursplaner och betygskriterier, Fritzes and Skolverket, Stockholm, 2000. 
sité d'Uppsala et approuvé par la commission de validation du Conseil en octobre 2001. Comme dans les anciens plans de cours, il est laissé une grande liberté à l'enseignant en matière de méthode de travail et d'organisation. Que l'élève atteigne les objectifs définis constitue la seule contrainte existante.

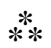

Alors que de nombreuses activités ont été organisées dans le cadre de l'Année européenne des langues 2001 et la Journée européenne des langues 2002, les jeunes Suédois semblent bouder l'apprentissage d'une seconde langue étrangère. La plupart des décideurs de l'éducation considèrent que la situation est inquiétante, non seulement au niveau scolaire, mais également universitaire tout comme en ce qui concerne l'éducation pour adultes. Il faut mentionner que l'enjeu économique a toujours été déterminant pour ce qui est de l'apprentissage obligatoire des langues dans un pays qui dépend largement de ses exportations de biens et de services (environ $45 \%$ du PNB). Très tôt, à cause de la petite taille du marché national, les entreprises industrielles suédoises se sont internationalisées ou sont devenues dépendantes d'autres pays. Il est également intéressant de noter que l'apprentissage obligatoire de deux langues étrangères nous ramène à un temps où ce dernier constituait une condition préalable pour les élèves suédois désirant poursuivre leurs études au lycée (ce n'est que depuis 1969 qu'ils ne sont plus obligés d'apprendre l'allemand ou le français pour entreprendre leurs études secondaires) et cela va sans dire, à l'université.

Enfin, alors que la diversité linguistique a été pendant des siècles considérée comme un désavantage et/ou un danger pour l'unité nationale, la Suède semble désireuse de s'affirmer comme pays plurilingue: en 2000, après s'être fait quelque peu prier, ce pays a enfin ratifié la Charte européenne des langues régionales ou minoritaires et a reconnu cinq langues minoritaires nationales. Et lorsque l'on lit de récents ouvrages suédois traitant de l'enseignement des langues étrangères, on est frappé de voir une partie consacrée au soutien des langues minoritaires. Toujours est-il que ce nouveau type de littérature dans laquelle sont dorénavant considérées dans leur ensemble toutes les langues en présence (étrangères et minoritaires, mais aussi le suédois langue maternelle et langue seconde) reflète la volonté d'instaurer une véritable politique linguistique. Celle-ci se trouve illustrée par le texte de proposition d'un programme d'action pour la langue suédoise rédigé par un comité parlementaire et publié l'année dernière ${ }^{20}$ visant à promouvoir le suédois en tant que langue principale en Suède (ce que jusqu'à présent la législation de ce pays n'a pas jugé utile de stipuler) pour, essentiellement, contre-attaquer l'influence grandissante de l'anglais, souvent considéré comme seconde langue dans l'environnement quotidien. Ce qui est intéressant ici, c'est que les auteurs ont effectué une étude

20. SOU 2002: 27, Handlingsprogram för svenska språket Mål i mun - Förslag till handlingsprogram för svenska språket. 
de la situation linguistique dans son ensemble et qu'ils recommandent le renforcement de l'enseignement de toutes les langues (étrangères, minoritaires et d'origine) dans l'ensemble du système éducatif. C'est donc désormais dans la perspective d'une politique linguistique que les décideurs suédois de l'éducation doivent trouver le moyen de surmonter le semi-échec de l'introduction de l'apprentissage obligatoire d'une seconde langue étrangère. Si des mesures pourraient être prises au niveau scolaire, comme la suppression de la possibilité d'abandonner cet apprentissage et la réévaluation des qualifications pour l'admission dans l'enseignement supérieur par la valorisation des compétences linguistiques des lycéens, le problème doit être posé au niveau de la société.

\section{BIBLIOGRAPHIE}

Languages - Syllabuses, grading criteria and comments, Gy2000, National Agency for Education and Fritzes, Stockholm, 2001.

CABAU-LAMPA B. (1998): L'enseignement des Langues - Cultures en Suède: un enjeu multidimensionnel, Université de Lille, Presses Universitaires du Septentrion.

Grundskoleförordning 1994:1194, Utbildningsdepartementet.

Grundskolans kursplaner och betygskriterier, Fritzes and Skolverket, Stockholm, 2000.

MALMBERG P. (2000): De moderna språken i grundskolan och gymnasieskolan från 1960 och framåt, Skolverket, Dnr 99:610, http://www.skolutveckling.se/ utvecklingsteman/sprak/filer/historik.pdf

Série de données statistiques Barnomsorg, skola och vuxenutbildning i siffror, Skolverket.

Skolverket, Nya kursplaner i språk, Nyhetsbrev, 20 december 1999, N²0.

Skolverket, Språkåret 2001- vad hände sedan?, Nyhetsbrev, 14 mai 2002, º5. SKOLVERKET (2002): Flera språk - fler möjligheter, Utveckling av odersmålsstödet och modersmålsundervisningen 2002, http://www.skolverket.se/ pdf/moders/huvud.pdf

SKOLVERKET (2002): Barnomsorg, skola och vuxenutbildning i siffror 2002: Del 2, Skolverkets rapport $\mathrm{N}^{\circ} 214$.

SKOLVERKET (2002): Beskrivande data om Barnomsorg, skola och vuxenutbildning 2002, Skolverkets rapport nr 218.

SÖRENSEN C. (2000): Språkvalet $i$ grundskolan - en pilotundersökning, Skolverket, Stockholm.

SOU (2002 : 27) : Handlingsprogram för svenska språket Mål i mun - Förslag till handlingsprogram för svenska språket. 


\section{L'organisation de l'enseignement des langues en Suède}

\section{Enseignement primaire}

La scolarité obligatoire (neuf années) englobe l'enseignement primaire. L'enseignement fondamental est dispensé en suédois sauf dans les classes bilingues ou dans les écoles privées à profil ethnique/linguistique.

Il existe six écoles publiques lapones offrant un enseignement couvrant les six premières années de scolarité. En 2001/2002, elles regroupaient 153 élèves, avec des cours de et en lapon et cours d'anglais (voir infra). Pour les enfants d'origine immigrée à l'école de base, l'enseignement se fait en langue maternelle. L'anglais reste une matière obligatoire dont l'apprentissage peut commencer dès la première année de scolarité, selon la décision de la commune. Des cours de suédois langue seconde sont proposés à tous les niveaux pour les enfants d'origine étrangère.

\section{Enseignement secondaire et professionnel}

L'enseignement des disciplines fondamentales est généralement assuré en suédois. Quelques cours de contenu sont proposés en langue étrangère dans le cadre du Programme SPRINT (Språk- och innehållsintegrerad inlärning och undervisning) correspondant au CLIL (Content and Language Integrated Learning, auquel il faudrait ajouter "Teaching»). Selon un rapport publié en $2000^{1}$, le programme est présent dans environ $4 \%$ des écoles de base et plus de $20 \%$ des lycées. Les langues-cibles associées au programme sont l'anglais $(75,5 \%)$, l'allemand, le français $(6 \%)$, l'espagnol $(4 \%)$, le finnois $(2 \%)$, autres $(4,5 \%)$.

L'enseignement de l'anglais est obligatoire. Il existe des classes bilingues (années 1-6) pour les élèves ayant l'anglais, le grec, le finnois ou l'espagnol comme langue maternelle. Pour ceux dont le finnois est la langue maternelle, un enseignement bilingue est assuré pendant les trois dernières années de l'école obligatoire.

\section{Enseignement public/privé}

L'enseignement varie complètement dans le cadre des écoles privées à profil ethnique/linguistique où l'accent est mis sur l'enseignement de la langue maternelle.

\section{Langues anciennes}

On les trouve seulement au niveau du lycée (grec et latin) dans quelques programmes. Quelques lycées privés proposent le latin en remplacement d'une seconde langue étrangère.

\section{Enseignement des langues régionales}

Le lapon et le finnois tornedalien sont proposés aux enfants dont la langue maternelle est le lapon ou le finnois de la vallée du Torne. A l'école de base, les élèves peuvent suivre des cours de langue maternelle dans les cadres suivants: à la place d'une seconde langue; en tant que choix de l'élève (soit 382 heures de cours réparties sur les neuf années de scolarité); dans le cadre des activités organisées par l'école (soit 600 heures maximum réparties sur l'ensemble de la scolarité) et enfin, en dehors de l'emploi du temps établi (pendant sept années maximum). Le nombre d'élèves minimum pour lequel les communes sont tenues d'ouvrir un cours en tant que langue B n'est pas fixé à cinq, comme cela est le cas pour les langues de l'immigration.

Il existe six écoles publiques lapones offrant un enseignement couvrant les six premières années de scolarité. En 2001/2002, elles regroupaient 153 élèves pour des cours de et en lapon, ainsi que des cours d'anglais. 


\section{Langues de l'immigration}

Par ordre d'importance à l'école de base: arabe, langues de l'ex-Yougoslavie (croate, serbe, bosniaque), finnois, espagnol, albanais, persan, anglais, turc, kurde, polonais. Enseignement offert en plus de cent vingt langues, sans compter celles mentionnées cidessus. Environ $12 \%$ des élèves de l'école de base ont droit à l'enseignement de leur langue maternelle. Un peu plus de la moitié d'entre eux y participent (contre $65 \%$ au début des années 1990).

Pour les élèves n'ayant pas le suédois comme langue usuelle quotidienne, les communes peuvent mettre en place un enseignement bilingue durant les six premières années de l'école de base et sur la totalité de la scolarité obligatoire si la langue en question est le finnois.

Il existe également des cours de soutien dont la langue d'enseignement est la langue maternelle. Quelques langues de l'immigration sont également enseignées dans les écoles privées à profil linguistique/ethnique.

A l'école de base, les élèves peuvent suivre des cours de langue maternelle dans les cadres suivants: à la place d'une seconde langue (possibilité très peu exploitée); en tant que choix de l'élève (soit 382 heures de cours réparties sur les neuf années de scolarité); dans le cadre des activités organisées par l'école (soit 600 heures maximum réparties sur l'ensemble de la scolarité) et enfin, en dehors de l'emploi du temps établi (pendant sept années maximum). Le nombre d'élèves minimum pour lequel les communes sont tenues d'ouvrir un cours en tant que langue $\mathrm{B}$ est fixé à cinq.

L'enseignement est dispensé soit dans une classe regroupant des élèves ayant la même langue maternelle, soit dans un groupe pour certaines matières (exemple: cours de mathématiques en espagnol). La plupart des communes organisent les cours en-dehors de l'emploi du temps, à raison d'une à deux heures par semaine. Possibilité de cours de langue maternelle au lycée.

\section{Expérimentations}

En 2001/2002, dans le secteur public, il y avait sept écoles de base et trois lycées à profil international; vingt-cinq lycées proposaient le programme du International Baccalaureat; dans le secteur privé, il y avait sept écoles de base internationales et trois lycées internationaux préparant au International Baccalaureat.

\section{Place des langues dans les certifications terminales}

Il existe au lycée une forme de certification spécifique en langue sous forme de tests nationaux dont la visée est de permettre aux enseignants de caler, d'étalonner leurs notes $^{2}$. Ces tests ne sont pas des examens, et si l'enseignant prend en considération le résultat, il n'existe pas de réglementation en matière de coefficient. A l'école de base, les élèves peuvent passer un test non obligatoire en anglais (matière principale) en cinquième année scolaire. En neuvième année, le test est obligatoire (Grundskoleförordningen $7 \mathrm{kap} .10 \$$ ).

\section{Grilles d'évaluation internationales}

Depuis 2000, le Cadre européen commun de référence du Conseil de l’Europe a fait son entrée dans le système éducatif suédois: les études de langues en sont profondément marquées et sont désormais organisées en niveaux (steg).

1. Nixon J. (2000), SPRINT Språk- och innehållsintegrerad inlärning och undervisning: Rapport på uppdrag av Skolverket, Skolverket, Stockholm.

2. Je tiens ici à remercier Michel Barbier du Département de la Formation continue de l'Université d'Uppsala pour les précisions apportées à ces deux questions. 